\title{
COMPARING IMPERFECTION RATIO AND IMPERFECTION INDEX FOR GRAPH CLASSES
}

\author{
Arie M.C.A. Koster ${ }^{1}$ And Annegret K. Wagler ${ }^{2}$
}

\begin{abstract}
Perfect graphs constitute a well-studied graph class with a rich structure, reflected by many characterizations with respect to different concepts. Perfect graphs are, for instance, precisely those graphs $G$ where the stable set polytope $\operatorname{STAB}(G)$ coincides with the fractional stable set polytope $\operatorname{QSTAB}(G)$. For all imperfect graphs $G$ it holds that $\operatorname{STAB}(G) \subset \operatorname{QSTAB}(G)$. It is, therefore, natural to use the difference between the two polytopes in order to decide how far an imperfect graph is away from being perfect. We discuss three different concepts, involving the facet set of $\operatorname{STAB}(G)$, the disjunctive index of $\operatorname{QSTAB}(G)$, and the dilation ratio of the two polytopes.

Including only certain types of facets for $\operatorname{STAB}(G)$, we obtain graphs that are in some sense close to perfect graphs, for example minimally imperfect graphs, and certain other classes of so-called rank-perfect graphs. The imperfection ratio has been introduced by Gerke and McDiarmid [12] as the dilation ratio of $\operatorname{STAB}(G)$ and $\operatorname{QSTAB}(G)$, whereas Aguilera et al. [1] suggest to take the disjunctive index of $\operatorname{QSTAB}(G)$ as the imperfection index of $G$. For both invariants there exist no general upper bounds, but there are bounds known for the imperfection ratio of several graph classes $[7,12]$.

Outgoing from a graph-theoretical interpretation of the imperfection index, we prove that there exists no upper bound on the imperfection index for those graph classes with a known bounded imperfection ratio. Comparing the two invariants on those classes, it seems that the imperfection index measures imperfection much more roughly than the imperfection ratio; we, therefore, discuss possible directions for refinements.
\end{abstract}

Keywords. Perfect graphs, imperfection ratio, imperfection index.

Mathematics Subject Classification. 05C17, 90C57.

Received May 24, 2007. Accepted January 09, 2008.

1 University of Warwick, Centre for Discrete Mathematics and its Applications (DIMAP), Coventry CV4 7AL, UK; Arie.Koster@wbs.ac.uk - Supported by the DFG research group "Algorithms, Structure, Randomness" (Grant number GR 883/9-3, GR 883/9-4).

2 Otto-von-Guericke-Universität Magdeburg, Fakultät für Mathematik, Institut für Mathematische Optimierung (IMO), Universitätsplatz 2, 39106 Magdeburg, Germany;

wagler@imo.math . uni-magdeburg.de 


\section{INTRODUCTION}

The stable set polytope $\operatorname{STAB}(G)$ of a graph $G=(V, E)$ is defined as the convex hull of the incidence vectors of all stable sets of $G$ (in a stable set all nodes are mutually nonadjacent). A canonical relaxation of $\operatorname{STAB}(G)$ is the fractional stable set polytope $\operatorname{QSTAB}(G)$ given by all "trivial" facets, the nonnegativity constraints $x_{i} \geq 0$ for all nodes $i$ of $G$, and by the clique constraints

$$
\sum_{i \in Q} x_{i} \leq 1
$$

for all cliques $Q \subseteq V$ (in a clique all nodes are mutually adjacent). We have $\operatorname{STAB}(G) \subseteq \operatorname{QSTAB}(G)$ for any graph but equality for perfect graphs only [6]. According to a famous characterization recently achieved by Chudnovsky et al. [5], that are precisely the graphs without chordless cycles $C_{2 k+1}$ with $k \geq 2$, termed odd holes, or their complements, the odd antiholes $\bar{C}_{2 k+1}$ (the complement $\bar{G}$ has the same nodes as $G$, but two nodes are adjacent in $\bar{G}$ iff they are non-adjacent in $G$ ). In particular, perfect graphs are closed under taking complements (Perfect Graph Theorem [16]). Perfect graphs turned out to be an interesting and important class with a rich structure and a nice algorithmic behaviour, see [19] for a recent survey. In particular, several parameters which are hard to evaluate in general can be determined in polynomial time if $G$ is perfect [13].

For all imperfect graphs $G$ it follows that $\operatorname{STAB}(G) \subset \operatorname{QSTAB}(G)$. It is natural to use the difference between the two polytopes in order to determine how far a certain imperfect graph is away from being perfect. We consider three ways to classify imperfect graphs: by description of $\operatorname{STAB}(G)$, the imperfection ratio, and the imperfection index.

Polytope descriptions. The first possibility is to extend the clique constraints describing $\operatorname{QSTAB}(G)$ to rank constraints

$$
\sum_{i \in V^{\prime}} x_{i} \leq \alpha\left(G^{\prime}\right)
$$

associated with arbitrary induced subgraphs $G^{\prime}=\left(V^{\prime}, E^{\prime}\right)$ in order to obtain $\operatorname{STAB}(G)$ (here, $\alpha\left(G^{\prime}\right)$ denotes the cardinality of a maximum stable set in $G^{\prime}$; we have $\alpha\left(G^{\prime}\right)=1$ iff $G^{\prime}$ is a clique and also write $(2)$ as $x\left(G^{\prime}, \mathbb{1 l}\right) \leq \alpha\left(G^{\prime}\right)$ ). That way, several well-known graph classes are defined: near-perfect graphs [20] where rank constraints associated with cliques and the whole graph are allowed only; t-perfect graphs [6] resp. h-perfect graphs [13] where rank constraints associated with edges, triangles, and odd holes resp. cliques of arbitrary size and odd holes are used only; and rank-perfect graphs [21] including the rank constraints associated with all induced subgraphs.

Further classes of rank-perfect graphs are line graphs [9] and antiwebs [22]. A line graph is obtained by taking the edges of a given graph as nodes and connecting two nodes iff the corresponding edges are incident. An antiweb $K_{n / k}$ is a graph 
with $n$ nodes $0, \ldots, n-1$ and edges $i j$ iff $k \leq|i-j| \leq n-k$ and $i \neq j$. Antiwebs include all cliques $K_{n}=K_{n / 1}$, all odd antiholes $\bar{C}_{2 k+1}=K_{2 k+1 / 2}$, and all odd holes $C_{2 k+1}=K_{2 k+1 / k}$. As common generalization of perfect, t-perfect, and hperfect graphs as well as antiwebs, the class of a-perfect graphs was introduced in [23] as those graphs whose stable set polytopes are given by nonnegativity constraints and rank constraints associated with antiwebs only.

Imperfection ratio. Gerke and McDiarmid [12] introduced the imperfection ratio $\operatorname{imp}(G)$ as the dilation ratio

$$
\operatorname{imp}(G)=\min \{t: \operatorname{QSTAB}(G) \subseteq t \operatorname{STAB}(G)\}
$$

of the two polytopes. We clearly have $\operatorname{imp}(G)=1$ iff $G$ is perfect and $\operatorname{imp}(G)>1$ iff $G$ is imperfect. Moreover, $\operatorname{imp}(G)=\operatorname{imp}(\bar{G})$ holds for all graphs [12]. The imperfection ratio is NP-hard to compute and unbounded in general [12]. So far, there are upper bounds known for the imperfection ratio of only some graph classes, including odd holes, t-perfect, h-perfect, and line graphs [12], antiwebs and a-perfect graphs [7] (and the corresponding complementary classes). We introduce two further graph classes and show that they have also a bounded imperfection ratio, see Section 2.

Imperfection index. Aguilera et al. [1] investigated the antiblocking duality of $\operatorname{STAB}(G)$ and $\operatorname{QSTAB}(G)$ by means of the disjunctive procedure introduced in [2] (see Sect. 3). They observed that the disjunctive index of $\operatorname{QSTAB}(G)$ can be seen as a measure of imperfection and suggest the imperfection index of $G$ as

$$
\operatorname{imp}_{\mathrm{I}}(G)=\min \left\{|J|: P_{J}(\operatorname{QSTAB}(G))=\operatorname{STAB}(G), J \subseteq V\right\}
$$

where $P_{J}(\operatorname{QSTAB}(G))=\operatorname{conv}\left\{x \in \operatorname{QSTAB}(G): x_{j} \in\{0,1\}, j \in J\right\}$.

We have $\operatorname{imp}_{\mathrm{I}}(G)=0$ iff $G$ is perfect and $\operatorname{imp}_{\mathrm{I}}(G)=1$ if $G$ is minimal imperfect (that is $G$ is not perfect but every proper induced subgraph is perfect). As precisely the graphs $G$ with $\operatorname{imp}_{\mathrm{I}}(G) \leq 1$, we introduce the class of almost-perfect graphs as those graphs $G$ which admit one node whose removal yields a perfect graph. This class clearly contains perfect and minimally impefect graphs, we present further examples. Moreover, it is proved in [1] that $\operatorname{imp}_{\mathrm{I}}(G)=\operatorname{imp}_{\mathrm{I}}(\bar{G})$ holds for all graphs. We present an alterative proof for this fact in terms of hypergraphs (Sect. 3).

The stable set problem can be solved in polynomial time for graphs with imperfection index bounded by a constant: we can solve $2^{\mathrm{imp}(G)}$ stable set problems on perfect graphs. In this perspective, it is unlikely to find many graph classes with bounded imperfection index. In fact, we show that for all known graph classes with bounded imperfection ratio, the imperfection index cannot be bounded, even for some of them where the stable set problem can be solved in polynomial time with the help of other approaches (e.g., t-perfect or line graphs). For this, we investigate the behaviour of the imperfection index by means of taking disjoint unions (Sect. 3), taking lexicographic products (Sect. 4), and substituting nodes 
by other graphs (Sect. 5). For the latter, we characterize how several classes of rank-perfect graphs behave under substitution.

Hence, our results suggest that the value of the imperfection index is a less intuitive measure on the closeness of the graph to perfectness than the imperfection ratio (see Sect. 6). Several suggestions for refinements conclude this paper.

\section{GRAPH Classes With BOUNDED IMPERFECTION RATIO}

Gerke and McDiarmid [12] introduced the imperfection ratio originally as

$$
\operatorname{imp}(G)=\max \left\{\frac{\chi_{f}(G, c)}{\omega(G, c)} \mid c: V(G) \rightarrow \mathbb{Z}_{+}\right\}
$$

i.e., as the maximum ratio of the fractional chromatic number and the clique number in their weighted versions, taken over all positive integral weight vectors.

There does not exist a general upper bound on the imperfection ratio due to the following reason. The so-called Mycielski graphs $G_{0}, G_{1}, G_{2}, \ldots$ form a famous series of graphs with $\omega\left(G_{i}\right)=2$ for all $i$, but $\chi\left(G_{i}\right)=2+i$ [17] (where $G_{0}=K_{2}$, $G_{1}=C_{5}$, and $G_{2}$ is the well-known Grötzsch graph). Larsen et al. [14] proved the unexpected recurrence $\chi_{f}\left(G_{i+1}\right)=\chi_{f}\left(G_{i}\right)+\frac{1}{\chi_{f}\left(G_{i}\right)}$. As $\operatorname{imp}(G)=\frac{\chi_{f}(G)}{2}$ holds for any triangle-free graph $G$ by [12], this implies

$$
\operatorname{imp}\left(G_{i}\right) \rightarrow \infty \text { for } i \rightarrow \infty
$$

and, thus, the Mycielski graphs $G_{0}, G_{1}, G_{2}, \ldots$ form a sequence with unbounded imperfection ratio.

However, there are also classes with bounded imperfection ratio. By [12], it holds that

$$
\operatorname{imp}(G)=\left\{\frac{2 k+1}{2 k}: C_{2 k+1} \text { shortest odd hole in } G\right\}
$$

whenever $G$ is a line graph or h-perfect and

$$
\operatorname{imp}(G)=\left\{\frac{2 k+1}{2 k}: 2 k+1 \text { length of shortest odd (anti)hole in } G\right\}
$$

for all co-h-perfect graphs $G$ where $\operatorname{STAB}(G)$ is given by rank constraints associated with cliques, odd holes, and odd antiholes only. As the $C_{5}$ is the shortest odd (anti)hole, this implies that $\operatorname{imp}(G) \leq \frac{5}{4}$ holds for all graphs $G$ belonging to one of these classes.

Note that odd (anti)holes are special partitionable graphs; that are graphs $G$ where, for any node $v$, the subgraph $G-v$ can be partitioned into $\alpha(G)$ cliques of maximum size $\omega(G)$ or into $\omega(G)$ stable sets of maximum size. We shall extend the above results to a common superclass of perfect, t-perfect, h-perfect, and co-hperfect graphs: we call a graph $G$ p-perfect if $\operatorname{STAB}(G)$ is given by rank constraints associated with cliques and partitionable subgraphs only. 
Theorem 2.1. For any imperfect p-perfect graph $G$, we have

$$
\operatorname{imp}(G)=\max \left\{\frac{\left|G^{\prime}\right|}{\left|G^{\prime}\right|-1}: G^{\prime} \subseteq G \text { minimally imperfect }\right\} .
$$

Proof. Consider a p-perfect graph $G$ having a partitionable graph $P$ as induced subgraph. By definition, it follows $|P|=\alpha^{\prime} \omega^{\prime}+1=n^{\prime}$. Consider a vector $x \in$ $\operatorname{QSTAB}(G)$. We have $x(P) \leq \frac{n^{\prime}}{\omega^{\prime}}$ as each node of $P$ can be covered $\omega^{\prime}$ times by the $n^{\prime}$ maximum cliques of $P$ by [3]. Let $y=\frac{\alpha^{\prime} \omega^{\prime}}{n^{\prime}} x$ (note that $y$ belongs to $\operatorname{QSTAB}(G)$ as $\left.\frac{\alpha^{\prime} \omega^{\prime}}{\alpha^{\prime} \omega^{\prime}+1}<1\right)$. Now,

$$
y(P)=\frac{\alpha^{\prime} \omega^{\prime}}{n^{\prime}} x(P) \leq \frac{\alpha^{\prime} \omega^{\prime}}{n^{\prime}} \frac{n^{\prime}}{\omega^{\prime}}=\alpha^{\prime}
$$

holds, and thus $y \in \operatorname{STAB}(G)$. It follows that $\operatorname{QSTAB}(G) \subseteq \frac{\alpha^{\prime} \omega^{\prime}+1}{\alpha^{\prime} \omega^{\prime}} \operatorname{STAB}(G)$ holds. This implies

$$
\operatorname{imp}(G)=\max \left\{\frac{\alpha^{\prime} \omega^{\prime}+1}{\alpha^{\prime} \omega^{\prime}}: P \subseteq G \text { partitionable }\right\}
$$

and, since any partitionable graph contains a minimally imperfect one, the partitionable subgraph $P \subseteq G$ with smallest cardinality (and, thus, maximal value $\left.\frac{|P|}{|P|-1}\right)$ is minimally imperfect.

As the $C_{5}$ is the smallest such partitionable graph, this implies $\operatorname{imp}(G) \leq \frac{5}{4}$ for the larger class of p-perfect graphs, too.

A similar result was shown in [7] for antiwebs, a-perfect graphs, and a further superclass of antiwebs, the near-bipartite graphs where the set of non-neighors of every node splits into two stable sets. According to [7], for all such graphs $G$,

$$
\operatorname{imp}(G)=\max \left\{\frac{n^{\prime}}{\alpha^{\prime} \omega^{\prime}}: K_{n^{\prime} / \alpha^{\prime}} \subseteq G\right\}
$$

where $\omega^{\prime}=\left\lfloor n^{\prime} / \alpha^{\prime}\right\rfloor$ holds and, in addition, the imperfection ratio of an antiweb is bounded by $\operatorname{imp}\left(K_{n / \alpha}\right)<\frac{3}{2}$. The complements of antiwebs are called webs, the complements of near-bipartite graphs are called quasi-line graphs (note that they contain all line graphs). By the invariance of the imperfection ratio under complementation, the imperfection ratio of any near-bipartite (resp. quasi-line) graph is, therefore, characterized by means of its induced antiwebs (resp. webs) only and is less than $\frac{3}{2}$.

In addition, Gerke and McDiarmid [12] showed that the imperfection ratio of planar graphs is bounded by $\frac{11}{6}$ (and conjectured that it is in fact bounded by $\frac{3}{2}$ ).

Finally, we present a (rough) bound on the imperfection ratio for the class of almost-perfect graphs:

Theorem 2.2. For any almost-perfect graph $G$, we have $\operatorname{imp}(G)<2$.

Proof. Let $v$ be a node such that $G-v$ is perfect. This implies $\chi(G-v, c)=$ $\chi_{f}(G-v, c)=\omega(G-v, c)$ for all weight vectors $c>0$. On the other hand, 
$\chi_{f}(G, c) \leq \chi(G, c) \leq \chi(G-v, c)+c_{v}$ and $\omega(G, c) \geq \max \left\{\omega(G-v, c), c_{v}+c_{u(c)}\right\}$ with $u(c)=\arg \max _{w \in N(v)} c_{w}$ holds, where $N(v)$ is the set of neighbors of $v$ in $G$. Thus

$$
\frac{\chi_{f}(G, c)}{\omega(G, c)} \leq \frac{\chi(G-v, c)+c_{v}}{\max \left\{\omega(G-v, c), c_{v}\right\}}=\frac{\chi(G-v, c)+c_{v}}{\max \left\{\chi(G-v, c), c_{v}+c_{u(c)}\right\}}<2
$$

holds for all $c>0$, which completes the proof.

\section{THE IMPERFECTION INDEX IN GRAPH THEORETICAL TERMS}

Balas et al. [2] introduced the disjunctive procedure for binary linear programs as a way to obtain a complete description of the integer polytope from the polytope described by the linear relaxation. Let $V=\{1, \ldots, n\}$ denote the set of binary variables. A run of the procedure for variable $x_{j}$ consists of three steps: (i) multiplication of the system of inequalities once by $x_{j}$ and once by $1-x_{j}$; (ii) linearization of the quadratic terms; and (iii) projection of the resulting system back to the $x$-space. Balas et al. [2] prove that the resulting polyhedron $P_{j}(X)$ equals $\operatorname{conv}\left\{x \in X: x_{j} \in\{0,1\}\right\}$. In fact, for a subset $J=\left\{i_{1}, \ldots, i_{j}\right\}$ of the variables,

$$
P_{J}(X)=\operatorname{conv}\left\{x \in X: x_{j} \in\{0,1\}, j \in J\right\}
$$

holds. Clearly, $P_{V}(X)=\operatorname{conv}\left(X \cap\{0,1\}^{n}\right)$, but also proper subsets can have this property. This result allows to define the disjunctive index of a polytope $X$ as the minimum size of a set $J \subseteq V$ such that $P_{J}(X)=\operatorname{conv}\left(X \cap\{0,1\}^{n}\right)$.

The imperfection index of a graph $G$ is defined as the disjunctive index of $\operatorname{QSTAB}(G)$. The following result directly follows from the definition. Here $G[V-j]$ denotes the subgraph of $G=(V, E)$ induced by $V \backslash\{j\}$.

Lemma $3.1([4])$. $P_{j}(\operatorname{QSTAB}(G))=\operatorname{STAB}(G)$ if and only if $G[V-j]$ is perfect.

This immediatly implies:

Corollary 3.2. $\operatorname{imp}_{\mathrm{I}}(G)=1$ if and only if there exists a node $j \in V$ such that $G[V-j]$ is perfect.

This shows in particular that the almost-perfect graphs are exactly those graphs $G$ with and imperfection index at most one (as they are defined to admit one node whose removal results in a perfect graph). Clearly, all perfect graphs $G$ are almost-perfect by $\operatorname{imp}_{\mathrm{I}}(G)=0$ as well as all minimally imperfect graphs $G$ by $\operatorname{imp}_{\mathrm{I}}(G)=1$ (note that in the latter graphs, removing any node yields a perfect graph). A subclass of t-perfect graphs, the almost-bipartite graphs, forms a further class with imperfection index at most one as they are defined to admit one node whose removal yields a bipartite graph.

Note that the class of almost-perfect graphs clearly contains graphs other than perfect, minimal imperfect, and almost-bipartite graphs, e.g., all odd wheels and odd antiwheels (the latter are obtained as complete join of an odd antihole and a single node). 
Lemma 3.1 can be generalized further as follows (this was independently observed in [18] and [15]).

Lemma $3.3([15,18]) . P_{J}(\operatorname{QSTAB}(G))=\operatorname{STAB}(G)$ if and only if $G[V-J]$ is perfect.

Therefore, if $P_{J}(\operatorname{QSTAB}(G))=\operatorname{STAB}(G), J$ is a subset of nodes meeting all minimal imperfect subgraphs of $G$. By the Perfect Graph Theorem [16], an induced subgraph $G^{\prime}$ of $G$ is minimally imperfect if and only if its complement $\bar{G}^{\prime}$ is minimally imperfect. Hence, the same node-subset $J$ meets all minimal imperfect subgraphs in the complementary graph, which implies:

Corollary 3.4 ([1]). Let $G=(V, E)$ be a graph. $P_{J}(\operatorname{QSTAB}(G))=\operatorname{STAB}(G)$ holds for a subset of nodes $J \subseteq V$ if and only if $P_{J}(\operatorname{QSTAB}(\bar{G}))=\operatorname{STAB}(\bar{G})$.

Hence, the imperfection index is invariant under taking complements, as shown before by Aguilera et al. [1].

We shall formalize the computation of the imperfection index further. For a graph $G=(V, E)$, we introduce the imperfection hypergraph $\mathcal{I}(G)=(V, \mathcal{F})$ on the same node set as $G$ and all node subsets inducing minimally imperfect subgraphs of $G$ as hyperedges. Obviously, we have $\mathcal{I}(G)=\mathcal{I}(\bar{G})$. For our purpose, we look for a minimum node cover of $\mathcal{I}(G)$, i.e., for a subset $J \subseteq V$ meeting all hyperedges. Obviously, any node cover of $\mathcal{I}(G)$ corresponds to a subset $J \subseteq V$ with $G[V-J]$ perfect resp. with $P_{J}(\operatorname{QSTAB}(G))=\operatorname{STAB}(G)$. This implies that the imperfection index of $G$ equals the node cover number $\tau(\mathcal{I}(G))$.

Lemma 3.5. For any graph $G, \operatorname{imp}_{\mathrm{I}}(G)=\operatorname{imp}_{\mathrm{I}}(\bar{G})=\tau(\mathcal{I}(G))=\tau(\mathcal{I}(\bar{G}))$.

From this graph-theoretical reformulation of $\operatorname{imp}_{\mathrm{I}}(G)$, we infer:

Lemma 3.6. The number of disjoint minimally imperfect subgraphs of $G$ is a lower bound on $\operatorname{imp}_{\mathrm{I}}(G)$.

Proof. Let $\mathcal{S}$ be a set of mutually disjoint subsets of $V$ that induce minimally imperfect subgraphs. For all $S \in \mathcal{S}$ we have to select at least one node in the node cover. Thus, $\tau(\mathcal{I}(G))$ is at least the size of $\mathcal{S}$.

Corollary 3.7. The imperfection index of a graph $G$ equals the sum of the imperfection indices of its maximal 2-edge-connected induced subgraphs.

Proof. Clearly, the imperfection index of a graph $G$ is the sum of the imperfection indices of its connected components (since the components of the hypergraph $\mathcal{I}(G)$ are defined on the same node subsets).

Now, let $G$ be a connected graph and $\mathcal{I}(G)$ the imperfection hypergraph as defined above. Moreover, let $e \in E$ be such that $G-e$ is not connected ( $e$ is a bridge). Since the only minimally imperfect subgraphs are odd holes and odd antiholes, $\mathcal{I}(G)$ is not connected. In fact, $\mathcal{I}(G)$ and $\mathcal{I}(G-e)$ are isomorphic. Hence, $\operatorname{imp}_{\mathrm{I}}(G)=\operatorname{imp}_{\mathrm{I}}(G-e)$ and the result follows by iteration for all bridges and application of the first part of the proof on the resulting non-connected graph. 
As a consequence, we obtain that the imperfection index cannot be bounded for several classes of graphs.

Theorem 3.8. For the following graph classes $\mathcal{G}$, there exists no upper bound on the imperfection index $\operatorname{imp}_{\mathrm{I}}(G), G \in \mathcal{G}:$ t-perfect graphs (and therefore, also $h$ perfect, p-perfect, a-perfect, rank-perfect graphs); line graphs (and therefore, also quasi-line graphs); planar graphs.

Proof. Let $k C_{5}$ be the disjoint union of $k$-holes. Then we obviously have $\operatorname{imp}_{\mathrm{I}}\left(k C_{5}\right)=k$ and, in particular,

$$
\operatorname{imp}_{\mathrm{I}}\left(k C_{5}\right) \rightarrow \infty \text { if } k \rightarrow \infty .
$$

As such graphs $k C_{5}, k \geq 1$ belong to the classes of t-perfect graphs as well as line graphs as well as planar graphs, the result follows for all these classes and their superclasses.

Similar constructions are possible by linking odd holes through additional edges to a chain; even in highly connected graphs many disjoint odd holes can occur:

Theorem 3.9. For the following graph classes $\mathcal{G}$, there exists no upper bound on the imperfection index $\operatorname{imp}_{\mathrm{I}}(G), G \in \mathcal{G}$ : webs and antiwebs (and therefore, also a-perfect, near-bipartite, and quasi-line graphs).

Proof. Let $\bar{K}_{5 k /(k+1)}$ be the web with $5 k$ nodes that is the complement of $K_{5 k /(k+1)}$. For $i \in\{1, \ldots, 5 k\}, \bar{K}_{5 k /(k+1)}$ contains the 5 -hole $C(i)=\{i, i+k, i+2 k, i+3 k, i+$ $4 k\}$. Hence, $\bar{K}_{5 k /(k+1)}$ contains $k$ disjoint 5 -holes $C(i)$ for $1 \leq i \leq k$. This implies that $\operatorname{imp}_{\mathrm{I}}\left(\bar{K}_{5 k /(k+1)}\right) \geq k$ and, in particular,

$$
\operatorname{imp}_{\mathrm{I}}\left(\bar{K}_{5 k /(k+1)}\right) \rightarrow \infty \text { if } k \rightarrow \infty \text {. }
$$

Thus, there is also no upper bound of the imperfection index for the classes of webs and antiwebs as well as for any of their superclasses.

\section{The IMPERFECTION INDEX AND LEXICOGRAPHIC PRODUCTS}

The lexicographic product $G_{1} \times G_{2}$ of two graphs $G_{1}$ and $G_{2}$ is obtained by substituting every node of $G_{1}$ by the graph $G_{2}$. Let $v$ be a node of a graph $G_{1}$ then substituting $v$ by another graph $G_{2}$ means to delete $v$ and to join every neighbor of $v$ in $G_{1}$ to every node of $G_{2}$. (Note that we exclude the two trivial cases if $G_{2}=\emptyset$ and if $v$ does not have any neighbor.)

Gerke and McDiarmid [12] studied the behavior of the imperfection ratio under taking lexicographic products $G_{1} \times G_{2}$ and showed that

$$
\operatorname{imp}\left(G_{1} \times G_{2}\right)=\operatorname{imp}\left(G_{1}\right) \cdot \operatorname{imp}\left(G_{2}\right)
$$


holds. Thus, the imperfection ratio cannot be bounded for any class $\mathcal{G}$ of graphs which is closed under substitution (and, therefore, closed under taking lexicographic products) and contains at least one imperfect graph $G$ as

$$
\operatorname{imp}\left(G^{i}\right) \rightarrow \infty \text { for } i \rightarrow \infty
$$

if $\operatorname{imp}(G)>1$ (where $G^{i}$ stands for $G \times \ldots \times G, i$ times). A necessary condition for a class $\mathcal{G}$ to have bounded imperfection ratio is, therefore, that $\mathcal{G}$ is closed under substituting perfect graphs for nodes only.

We consider the behavior of the imperfection index under taking lexicographic products $G_{1} \times G_{2}$ as well.

Theorem 4.1. For two graphs $G_{1}, G_{2}$ we have

$$
\operatorname{imp}_{\mathrm{I}}\left(G_{1} \times G_{2}\right)=\left|G_{2}\right| \operatorname{imp}_{\mathrm{I}}\left(G_{1}\right)+\left(\left|G_{1}\right|-\operatorname{imp}_{\mathrm{I}}\left(G_{1}\right)\right) \cdot \operatorname{imp}_{\mathrm{I}}\left(G_{2}\right) .
$$

Proof. Let $W$ be an arbitrary set of nodes in $G_{1} \times G_{2}$ that meets each imperfect subgraph. Let $V_{1}^{\prime}$ be the subset of nodes of $G_{1}=\left(V_{1}, E_{1}\right)$ such that $W$ contains all of the substitutes of $v$ in $G_{1} \times G_{2}$, and let $U$ be the subset of $W$ for which not all substitutes of a node in $G_{1}$ are included. Suppose $V_{1}^{\prime}$ does not meet all imperfect graphs of $G_{1}$. Let $S_{1} \subseteq V_{1}$ induce such an unmet imperfect subgraph. Define $S$ by selecting one substitute $v \notin W$ for every node in $S_{1}$. The subgraph of $G_{1} \times G_{2}$ induced by $S$ is isomorphic to $G_{1}[S]$ and hence imperfect. A contradiction and thus $|W-U| \geq\left|G_{2}\right| \operatorname{imp}_{\mathrm{I}}\left(G_{1}\right)$.

Now consider a node $v \in V_{1}-V_{1}^{\prime}$. The subgraph of $G_{1} \times G_{2}$ induced by the substitutes of $v$ is isomorphic with $G_{2}$ and thus at least $\operatorname{imp}_{\mathrm{I}}\left(G_{2}\right)$ nodes have to be included in $U$. We conclude

$$
|W| \geq\left|G_{2}\right| \operatorname{imp}_{\mathrm{I}}\left(G_{1}\right)+\left(\left|G_{1}\right|-\operatorname{imp}_{\mathrm{I}}\left(G_{1}\right)\right) \operatorname{imp}_{\mathrm{I}}\left(G_{2}\right)
$$

accordingly.

To bound $\operatorname{imp}_{\mathrm{I}}\left(G_{1} \times G_{2}\right)$ from above, define $W$ as follows. Let $V_{1}^{\prime} \subseteq V_{1}$ be a minimum node subset of $G_{1}=\left(V_{1}, E_{1}\right)$ such that $G_{1}\left[V_{1}-V_{1}^{\prime}\right]$ is perfect. Similarly, let $V_{2}^{\prime} \subseteq V_{2}$ be a minimum node subset of $G_{2}=\left(V_{2}, E_{2}\right)$ such that $G_{2}\left[V_{2}-V_{2}^{\prime}\right]$ is perfect. For $v \in V_{1}^{\prime}$, let $W$ contain all substitutes, whereas for $v \notin V_{1}^{\prime}$, let $W$ contain only the substitutes isomorphic to $V_{2}^{\prime}$. Hence, $W$ is of the desired size. Now, the graph $G_{1} \times G_{2}-W$ is isomorphic with $\left(G_{1}-V_{1}^{\prime}\right) \times\left(G_{2}-V_{2}^{\prime}\right)$, and hence $G_{1} \times G_{2}-W$ is perfect (since the lexicographic product of two perfect graphs is perfect again [16]).

Thus, also the imperfection index cannot be bounded for any class $\mathcal{G}$ of graphs which is closed under substitution (and, therefore, closed under taking lexicographic products) and contains at least one imperfect graph $G$. In contrary to the imperfection ratio, we have even more:

Corollary 4.2. Let $G_{1}$ be a graph. For any perfect graph $G_{2}$, we have

$$
\operatorname{imp}_{\mathrm{I}}\left(G_{1} \times G_{2}\right)=\left|G_{2}\right| \operatorname{imp}_{\mathrm{I}}\left(G_{1}\right) .
$$


As this result clearly also applies to the two special cases, namely taking lexicographic products where $G_{2}$ is a clique (replicating every node of $G_{1}$ ) or a stable set (multiplying every node of $G_{1}$ ), we immediatly obtain the following:

Corollary 4.3. Let $\mathcal{G}$ be a graph class containing one imperfect graph. If $\mathcal{G}$ is closed under substituting perfect graphs for nodes, replication, or multiplication, then there exists no upper bound for the imperfection index $\operatorname{imp}_{\mathrm{I}}(G), G \in \mathcal{G}$.

Thus, a sufficient condition for the non-existence of an upper bound on the imperfection index is that the graph class $\mathcal{G}$ in question contains an imperfect graph and is closed under substituting certain perfect graphs, whereas a necessary condition for the existence of an upper bound on the imperfection ratio for $\mathcal{G}$ is that $\mathcal{G}$ is closed under substituting perfect graphs for nodes only.

\section{Classes of Rank-Perfect Graphs and substitution}

The results from the previous section motivate to study the behaviour of the remaining graph classes of interest under substitution. So far, there are no bounds known on the imperfection ratio or the imperfection index of near-perfect and general rank-perfect graphs. On the one hand, we shall check whether these classes are closed under substituting certain perfect graphs; on the other hand, we shall ensure that substitution of imperfect graphs is not possible. This suggests to characterize what happens to these classes under substitution. Note that such a characterization gives, in addition, also some insight in how to construct graphs in the corresponding classes. This is of particular interest, as none of the subclasses of rank-perfect graphs is characterized in graph-theoretical terms yet (but only in polyhedral terms by means of the facets of the stable set polytope). Thus, we shall also address the behavior of h-perfect, co-h-perfect, p-perfect, and a-perfect graphs under substitution.

For our purpose, we shall make use of the following result:

Theorem 5.1. [6,8] Let $G$ be obtained by substituting a node $v$ of a graph $G_{1}=$ $\left(V_{1}, E_{1}\right)$ by a graph $G_{2}=\left(V_{2}, E_{2}\right)$. Then a non-trivial inequality is facet-defining for $\operatorname{STAB}(G)$ if and only if it can be scaled to be a facet product of the form

$$
\sum_{i \in V_{1}-v} a_{i}^{1} x_{i}+a_{v}^{1} \sum_{j \in V_{2}} a_{j}^{2} x_{j} \leq 1
$$

where $x\left(G_{i}, a^{i}\right) \leq 1$ is a non-trivial facet of $\operatorname{STAB}\left(G_{i}\right)$ for $i=1,2$.

Note that Chvátal [6] gave a linear description of $\operatorname{STAB}(G)$ outgoing from the stable set polytopes of the original graphs, whereas Cunningham [8] proved later that each of the inequalities found by Chvátal is indeed facet-defining. We study the consequences of this theorem for several subclasses of rank-perfect graphs. Throughout this section, all non-trivial inequalities are scaled to have right hand 
side equal to 1 (that means: only clique constraints keep unchanged, rank constraints $x\left(G^{\prime}, \mathbb{1}\right) \leq \alpha\left(G^{\prime}\right)$ turn to $x\left(G^{\prime}, a\right) \leq 1$ with $a=\left(\frac{1}{\alpha\left(G^{\prime}\right)}, \ldots, \frac{1}{\alpha\left(G^{\prime}\right)}\right)$, and non-rank constraints have different non-zero coefficients).

Proposition 5.2. Consider a graph $G$ obtained by substituting a node $v$ of a graph $G_{1}$ by $G_{2}$. If there is a non-trivial, non-clique facet of $\operatorname{STAB}\left(G_{2}\right)$ then $\operatorname{STAB}(G)$ has a non-trivial, non-rank facet.

Proof. Let $G_{1}=\left(V_{1}, E_{1}\right)$ and $G_{2}=\left(V_{2}, E_{2}\right)$ and take the facet product

$$
\sum_{i \in Q-v} x_{i}+\sum_{j \in V_{2}} a_{j}^{2} x_{j} \leq 1
$$

of a clique facet associated with $Q \subseteq V_{1}, v \in Q$ and a non-trivial, non-clique facet $x\left(G_{2}, a^{2}\right) \leq 1$ of $\operatorname{STAB}\left(G_{2}\right)$. Then there is a node $k \in V_{2}$ with $0<a_{k}^{2}<1$ and the above facet product has different non-zero coeffients: every $i \in Q-v$ has coefficient 1 but $0<a_{k}^{2}<1$ (recall: we exclude the case that $v$ does not have any neighbor, hence there is a clique $Q \subseteq V_{1}$ with $Q-v \neq \emptyset$ ). Thus, the above facet product is a non-trivial, non-rank facet of $\operatorname{STAB}(G)$.

That means, whenever $G_{2}$ is imperfect, the graph obtained by substituting $G_{2}$ for a node cannot be rank-perfect. Hence, none of the classes of rank-perfect graphs (different from the class of perfect graphs) is closed under substitution. In addition, we are interested which graphs $G_{1}$ and $G_{2}$ are allowed in order to produce a rank-perfect graph $G$ by substitution.

Theorem 5.3. Let $G$ be obtained by substituting a node $v$ of $G_{1}$ by $G_{2}$. $G$ is rank-perfect if and only if $G_{1}$ is rank-perfect and $G_{2}$ is perfect.

Proof. Let $G_{1}=\left(V_{1}, E_{1}\right)$ and $G_{2}=\left(V_{2}, E_{2}\right)$. Assume first that $G_{1}$ is rank-perfect and $G_{2}$ is perfect. Then $\operatorname{STAB}\left(G_{1}\right)$ admits only non-trivial facets $x\left(G_{1}, a^{1}\right) \leq 1$ with $a_{i}^{1} \in\{0, c\}$. Each facet product

$$
\sum_{i \in V_{1}-v} a_{i}^{1} x_{i}+a_{v}^{1} \sum_{j \in Q} x_{j} \leq 1
$$

of $x\left(G_{1}, a^{1}\right) \leq 1$ with an arbitrary clique facet associated with $Q \subseteq V_{2}$ has again $a_{i}^{1} \in\{0, c\}$ as only coefficients. Thus, the only non-trivial facets of $\operatorname{STAB}(G)$ are rank constraints.

Conversely, if $G$ is supposed to be rank-perfect then $G_{2}$ has to be perfect (otherwise $\operatorname{STAB}\left(G_{2}\right)$ has a non-trivial facet different from a clique constraint and $\operatorname{STAB}(G)$ has a non-rank facet by Prop. 5.2). $G_{1}$ has to be rank-perfect (otherwise $\operatorname{STAB}\left(G_{1}\right)$ has a non-trivial, non-clique facet and its facet product with an arbitrary clique facet of $\operatorname{STAB}\left(G_{2}\right)$ yields a non-trivial, non-clique facet of $\operatorname{STAB}(G))$.

Thus, precisely substituting perfect graphs for nodes preserves rank-perfectness and substituting imperfect graphs for nodes in near-perfect, h-perfect, a-perfect, 
or p-perfect graphs cannot preserve the membership in those classes, too. We are interested whether there are further requirements in order to obtain graphs belonging to one of these classes by substitution.

Note that Shepherd [20] showed that the class of near-perfect graphs is closed under replication (i.e., the special case of substitution where $G_{2}$ is a clique). We ensure that there is no other way to produce a near-perfect graph by substitution.

Theorem 5.4. Let $G$ be obtained by substituting a node $v$ of $G_{1}$ by $G_{2} . G$ is near-perfect if and only if either $G_{1}$ and $G_{2}$ are perfect or $G_{1}$ is near-perfect and $G_{2}$ is a clique.

Proof. The if-part follows from Shepherd [20], thus we only have to treat the only if-part. Let $G_{1}=\left(V_{1}, E_{1}\right)$ and $G_{2}=\left(V_{2}, E_{2}\right)$. Clearly, if $G$ is supposed to be perfect then $G_{1}$ and $G_{2}$ have to be perfect due to $G_{1}, G_{2} \subseteq G$. Hence assume that $G$ is near-perfect and imperfect. Then $G_{2}$ has to be perfect, otherwise $\operatorname{STAB}\left(G_{2}\right)$ has a non-trivial facet different from a clique constraint and $G$ is not rank-perfect by Proposition 5.2. $G$ imperfect and $G_{2}$ perfect implies $G_{1}$ imperfect, hence $\operatorname{STAB}\left(G_{1}\right)$ has a non-trivial, non-clique facet $x\left(G_{1}, a^{1}\right) \leq 1$. In particular, there is a node $k \in V_{1}$ with $0<a_{k}^{1}<1$. Consider the facet product

$$
\sum_{i \in V_{1}-v} a_{i}^{1} x_{i}+a_{v}^{1} \sum_{j \in Q} x_{j} \leq 1
$$

of $x\left(G_{1}, a^{1}\right) \leq 1$ with an arbitrary clique facet associated with $Q \subseteq V_{2}$. Then the facet product is a non-trivial, non-clique facet of $\operatorname{STAB}(G)$ by $0<a_{k}^{1}<1$ and, thus, the full rank facet as $G$ is near-perfect. Therefore, all coefficients are equal to $\frac{1}{\alpha(G)}$ and $a_{i}^{1}=\frac{1}{\alpha(G)}$ for all $i \in V_{1}$ and $Q=V_{2}$ follows. Hence, $x\left(G_{1}, a^{1}\right) \leq 1$ is the full rank facet of $\operatorname{STAB}\left(G_{1}\right)$ (note $\alpha(G)=\alpha\left(G_{1}\right)$ by $Q=V_{2}$ ) and its only non-trivial facet different from a clique constraint, and $G_{2}$ is a clique.

Finally, we also address the behavior of the remaining subclass of rank-perfect graphs under substitution. We obtain the following result for p-perfect graphs:

Theorem 5.5. Let $G$ be obtained by substituting a node $v$ of $G_{1}$ by $G_{2} . G$ is p-perfect if and only if $G_{1}$ is p-perfect and either $v$ is not contained in any partitionable subgraph of $G_{1}$ and $G_{2}$ is perfect or $v$ is contained in a partitionable subgraph of $G_{1}$ and $G_{2}$ is a stable set.

Proof. Let $G_{1}=\left(V_{1}, E_{1}\right)$ and $G_{2}=\left(V_{2}, E_{2}\right)$. Assume first $G_{1}$ to be p-perfect. If $v$ is not contained in any partitionable subgraph $P$ of $G_{1}$ and $G_{2}$ is perfect, then $\operatorname{STAB}(G)$ has besides facets of $\operatorname{STAB}\left(G_{1}\right)$ with vanishing coefficient for $v$ only products of trivial or clique facets, hence $G$ is p-perfect. If $G_{2}$ is a stable set, the assertion follows since multiplication preserves p-perfectness: if $G_{2}$ is stable then all non-trivial facets of $\operatorname{STAB}\left(G_{2}\right)$ are clique constraints associated with a single node and all facet products $(3)$ of $\operatorname{STAB}(G)$ are obtained by simply replacing $v$ by a node of $G_{2}$ (i.e., $\operatorname{STAB}(G)$ contains $\left|G_{2}\right|$ copies of every facet $x\left(G_{1}, a^{1}\right) \leq 1$ of $\operatorname{STAB}\left(G_{1}\right)$ with $\left.a_{v}^{1} \neq 0\right)$. 
Now, suppose $G$ to be p-perfect. Then $G_{2}$ is perfect by Proposition 5.2 (otherwise $G$ is even not rank-perfect). Consider the facet product

$$
\sum_{i \in V_{1}-v} a_{i}^{1} x_{i}+a_{v}^{1} \sum_{j \in Q} x_{j} \leq 1
$$

of an arbitrary non-trivial facet $x\left(G_{1}, a^{1}\right) \leq 1$ of $\operatorname{STAB}\left(G_{1}\right)$ and a clique facet associated with $Q \subseteq V_{2}$. Since $G$ is p-perfect, every facet product is either a clique constraint (then $x\left(G_{1}, a^{1}\right) \leq 1$ is a clique facet) or a rank constraint associated with a partitionable subgraph $P$ (then $x\left(G_{1}, a^{1}\right) \leq 1$ is the facet associated with $P$ with either $a_{v}^{1}=0$ or $a_{v}^{1} \neq 0$ and $\left.|Q|=1\right)$. That means: $G_{1}$ is p-perfect and, if $v$ is contained in a partitionable subgraph of $G_{1}$, then $G_{2}$ is a stable set.

The latter result includes the classes of h-perfect and co-h-perfect graphs (as odd holes and odd antiholes are special partitionable graphs). A similar argumentation applies to all a-perfect graphs (since the facet-defining antiwebs play the same role in a-perfect graphs as the partitionable subgraphs in p-perfect graphs). In particular, taking lexicographic products with stable sets preserves the membership in all those classes. Thus, we can summarize the results from this section as follows (the last point gives an alternative proof for assertions of Th. 3.8):

Corollary 5.6. There exists no upper bound for the imperfection index of the following graph classes:

- rank-perfect graphs (closed under substituting perfect graphs for nodes);

- near-perfect graphs (closed under replication);

- h-perfect, co-h-perfect, p-perfect, a-perfect graphs (closed under multiplication).

\section{CONCLUding REMARKS}

In this paper, we have studied three different ways to classify imperfect graphs according to their closeness to perfect graphs. Several classes of graphs are defined by their limited number of classes of facet defining inequalities different from trivial and clique inequalities. The imperfection ratio has been shown to be bounded for p-perfect graphs in this paper and for several other classes in previous papers. The imperfection index has been shown to be unbounded for all those classes for which the imperfection ratio has been shown to be bounded, $c f$. Tabel 1 which gives an overview of the results achieved.

An open question is whether there exist a graph class such that the imperfection index of all members is bounded by a constant $k$ with $1<k<\infty$ (other than perfect graphs and those defined by a constant). The stable set problem could be solved in polynomial time on such classes straightforward.

Since the imperfection index cannot be bounded even for classes of graphs where the stable set problem can be solved in polynomial time (e.g., t-perfect or line graphs), it is fair to conclude that the disjunctive index of $\operatorname{QSTAB}(G)$ is a too rough measure for intuitively determining the closeness of a graph $G$ to the class 
TABLE 1. Summary of the bounds.

\begin{tabular}{l|c|c}
\hline Graph class $\mathcal{G}$ & $\sup \{\operatorname{imp}(G): G \in \mathcal{G}\}$ & $\sup \left\{\operatorname{imp}_{\mathrm{I}}(G): G \in \mathcal{G}\right\}$ \\
\hline \hline perfect & $\leq 1$ & $=0$ \\
\hline minimal imperfect & $\leq \frac{5}{4}$ & $\leq 1$ \\
almost-bipartite & $\leq \frac{5}{4}$ & $\leq 1$ \\
almost-perfect & $\leq \frac{5}{4}$ & $\infty$ \\
\hline t-perfect & $\leq \frac{5}{4}$ & $\infty$ \\
h-perfect & $\leq \frac{5}{4}$ & $\infty$ \\
p-perfect & $\leq \frac{5}{4}$ & $\infty$ \\
line & $<\frac{3}{2}$ & $\infty$ \\
\hline antiwebs/webs & $<\frac{3}{2}$ & $\infty$ \\
a-perfect & $<\frac{3}{2}$ & $\infty$ \\
near-bipartite & $<\frac{3}{2}$ & $\infty$ \\
quasi-line & $\leq \frac{11}{6}$ & $\infty$ \\
\hline planar & $? ?$ & $\infty$ \\
\hline near-perfect & $? ?$ & $\infty$ \\
rank-perfect & $\infty$ & $\infty$ \\
\hline general & & \\
\hline & & $\infty$ \\
\hline
\end{tabular}

of perfect graphs. Possible refinements could be obtained as follows:

- The imperfection index can be redefined for (2-)connected graphs only. In this way the $k C_{5}$ example cannot be taken anymore. However, the odd holes can be connected with each other without loss of generality, and thus the new imperfection index would still not be bounded for all the above graph classes.

- The disjunctive procedure can be carried out with any linear combination $\pi x$ of the variables and a value $\pi_{0}$. The resulting polytope is then defined as

$$
P_{\left(\pi, \pi_{0}\right)}(X)=\operatorname{conv}\left(\left\{x \in X: \pi x \leq \pi_{0}\right\} \cup\left\{x \in X: \pi x \geq \pi_{0}+1\right\}\right) .
$$

For any near-perfect graph $G$ and (2) with $V^{\prime}=V$ and $\pi_{0}=\alpha(G)$ as $\pi x \leq \pi_{0}$, it directly follows $P_{\left(\pi, \pi_{0}\right)}(\mathrm{QSTAB}(G))=\operatorname{STAB}(G)$. If we define the genearalized disjunctive index to be equal to the minimum number of times the procedure have to be applied before obtaining $\operatorname{STAB}(G)$, the generalized disjunctive index would equal one in this case. The right choice of the pairs $\left(\pi, \pi_{0}\right)$ is however unclear in general. Moreover, $k C_{5}$ still needs $k$ applications of the disjunctive procedure before $\operatorname{STAB}(G)$ is reached. 
- The unboundedness of the imperfection index for classes of graphs bases in all the above cases on the increase of the number of nodes in the graph without leaving the class (disjoint union, substitution, replication, multiplication). Scaling the imperfection index by the number of nodes $n=|V|$ could resolve this problem.

We, therefore, suggest to consider the normalized imperfection index

$$
\operatorname{imp}_{\mathrm{n}}(G)=\frac{\operatorname{imp}_{\mathrm{I}}(G)}{n} .
$$

As there are no imperfect graphs with four or less nodes, $\operatorname{imp}_{\mathrm{I}}(G)$ can be at most $n-4$, and thus scaling yields a value $\operatorname{imp}_{n}(G) \in[0,1)$.

All perfect graphs are exactly the graphs with $\operatorname{imp}_{n}(G)=0$; all almost-perfect graphs satisfy $\operatorname{imp}_{\mathrm{n}}(G) \leq \frac{1}{n}$. Even for $k C_{5}, k \geq 1$, we obtain as normalized imperfection index $\frac{\operatorname{imp}_{\mathrm{I}}\left(k C_{5}\right)}{5 k}=0.2$, independent of $k$. Taking the lexicographic product of $k 5$-holes yields a sequence with

$$
\frac{\operatorname{imp}_{\mathrm{I}}\left(\left(C_{5}\right)^{k}\right)}{\left|\left(C_{5}\right)^{k}\right|} \rightarrow 1 \text { if } k \rightarrow \infty
$$

(since $\operatorname{imp}_{\mathrm{I}}\left(\left(C_{5}\right)^{k}\right)=5^{k}-4^{k}$ whereas $\left|\left(C_{5}\right)^{k}\right|=5^{k}$ ), which is consistant with the fact that also the imperfection ratios of these graphs tend to infinity. It is, however, interesting to observe that for the Mycielski graphs $G_{0}, G_{1}, G_{2}, \ldots$ the quotient of imperfection index and number of nodes tends to $\frac{1}{3}$, whereas their imperfection ratios cannot be bounded.

Acknowledgements. The authors would like to thank the referees for the valuable comments to improve the presentation of the results.

\section{REFERENCES}

[1] N.E. Aguilera, M.S. Escalante, and G.L. Nasini, A generalization of the Perfect Graph Theorem under the disjunctive index. Math. Oper. Res. 27 (2002) 460-469.

[2] E. Balas, G. Cornuéjols, and S. Ceria, A lift-and-project cutting plane algorithm for mixed 0-1 programs. Math. Program. 58 (1993) 295-324.

[3] R.G. Bland, H.-C. Huang, and L.E. Trotter, Jr., Graphical Properties Related to Minimal Imperfection. Discrete Math. 27 (1979) 11-22.

[4] S. Ceria, Lift-and-project cuts and perfect graphs. Math. Program. 98 (2003) 309-317.

[5] M. Chudnovsky, N. Robertson, P. Seymour, and R. Thomas, The Strong Perfect Graph Theorem. Ann. Math. 164 (2006) 51-229.

[6] V. Chvátal, On Certain Polytopes Associated with Graphs. J. Comb. Theory (B) 18 (1975) $138-154$.

[7] S. Coulonges, A. Pêcher, and A. Wagler, Characterizing and bounding the imperfection ratio for some graph classes. Math. Program. (to appear).

[8] W.H. Cunningham, Polyhedra for composed independence systems, in: Bonn Workshop on Combinatorial Optimization, edited by A. Bachem, M. Grötschel, and B. Korte, Ann. Discrete Math. 16 (1982) 57-67. 
[9] J.R. Edmonds, Maximum Matching and a Polyhedron with $(0,1)$ Vertices. J. Res. Nat. Bur. Standards 69B (1965) 125-130.

[10] A.M.H. Gerards and A. Schrijver, Matrices with the Edmonds-Johnson Property. Combinatorica 6 (1986) 403-417.

[11] A.M.H. Gerards, G. Maróti, and A. Schrijver, Note on: N.E. Aguilera, M.S. Escalante, and G.L. Nasini, "A generalization of the Perfect Graph Theorem under the disjunctive index". Math. Oper. Res. 28 (2003) 884-885.

[12] S. Gerke and C. McDiarmid, Graph imperfection I, II. J. Comb. Theor. (B) 83 (2001) 58-78, $79-101$.

[13] M. Grötschel, L. Lovász, and A. Schrijver, Geometric algorithms and combinatorial optimization. Springer-Verlag (1988).

[14] M. Larsen, J. Propp, and D.H. Ullman, The fractional chromatic number of Mycielski graphs. J. Graph Theory 20 (1995) 411-416.

[15] L. Lipták and L. Tunçel, Lift-and-project ranks and antiblocker duality. Oper. Res. Lett. 33 (2005) 35-41.

[16] L. Lovász, Normal hypergraphs and the weak perfect graph conjecture. Discrete Math. 2 (1972) 253-267.

[17] J. Mycielski, Sur le coloriage des graphs. Colloq. Math. 3 (1955) 161-162.

[18] G.L. Nasini, El índice de imperfección de un grafo y su complemento, in Anales de las XXX JAIIO-SIO'01 (2001) 101-106.

[19] B. Reed and J. Ramirez-Alfonsin, Perfect Graphs, Wiley (2001).

[20] F.B. Shepherd, Near-Perfect Matrices. Math. Program. 64 (1994) 295-323.

[21] A. Wagler, Relaxing perfectness: which graphs are "almost" perfect? in The sharpest cut, impact of Manfred Padberg and his Work, edited by M. Grötschel , MPS/SIAM series on Optimization 4 (2004).

[22] A. Wagler, Antiwebs are rank-perfect. A Quaterly Journal of Operations Research 2 (2004) $149-152$.

[23] A. Wagler, On the stable set polytopes of classes of near-bipartite graphs. A Quaterly Journal of Operations Research 3 (2005) 329-336. 